\title{
Design of Visual Communication Effect Evaluation Method of Artworks Based on Machine Learning
}

\author{
Hang Li ${ }^{1},{ }^{1}$ Ran Liu, ${ }^{1}$ Lu Wang, ${ }^{2}$ and Jie Zhang ${ }^{1}$ \\ ${ }^{1}$ School of Mechanical Engineering, Anyang Institute of Technology, Anyang, Henan 455000, China \\ ${ }^{2}$ Academy of Fine Arts, Anyang Normal University, Anyang, Henan 455000, China \\ Correspondence should be addressed to Hang Li; 20160290@ayit.edu.cn
}

Received 31 December 2021; Accepted 25 January 2022; Published 24 February 2022

Academic Editor: Hasan Ali Khattak

Copyright $\odot 2022$ Hang Li et al. This is an open access article distributed under the Creative Commons Attribution License, which permits unrestricted use, distribution, and reproduction in any medium, provided the original work is properly cited.

With the rapid development of computer and digital media technology, more and more visual artworks are created, preserved, and transmitted digitally, which has become an indispensable spiritual wealth in this era. However, the aesthetic evaluation of traditional visual artworks can only rely on artists or appreciation experts for qualitative description, and the results have great subjective uncertainty due to their different personal knowledge and experience. Therefore, computational aesthetics came into being, that is, using a computer model to assist in quantitative evaluation of visual artworks. These models have broad application prospects in the fields of aesthetic evaluation and correction, art style identification, and so on. Based on this, this paper proposes a design of visual communication effect evaluation method of artworks based on machine learning. First, some characteristic variables are constructed to quantify the balance, contrast, and harmony in icon design criteria, and these three common design criteria are transformed into a mathematical expression. Based on the powerful fitting, classification, and generalization capabilities of the SVM method, we choose it as our base model. Then, the artificial evaluation scores are regressed to the calculated characteristic variables to obtain the statistical linear regression models corresponding to the three design criteria. The experimental results show that the evaluation model and manual evaluation results can reach a significant correlation in the same dimension, which verifies the effectiveness of the model.

\section{Introduction}

Visual art refers to the use of certain material materials to transform human emotions and understanding of the world into intuitive visual images in people's sight, including twodimensional planar works, three-dimensional sculpture, dynamic film, and television [1]. From the beginning of human culture to today, human beings convey information through the visual images they create, which have a profound impact on the inheritance of human history and culture and spiritual life. Aesthetics is a science that studies aesthetic categories such as beauty and ugliness, people's aesthetic consciousness, aesthetic experience, as well as the creation, development, and laws of beauty with visual art as its main object [2]. The study of aesthetics has spanned philosophy, neuroscience, psychology, computer science, and other fields. The whole development process has spanned nearly 2,000 years $[3,4]$. However, the traditional aesthetic analysis of visual art can only rely on artists or appreciation experts to make qualitative descriptions according to their personal knowledge and opinions, and the results are highly dependent on the expert level and have subjective uncertainty [5]. The computer, as a general digital processing machine, can assist people to objectively count out some aesthetic or style characteristics of artistic works, so the use of computer-assisted human aesthetic analysis of artistic works is a perfect complement to the artistic aesthetic simplicity.

With the rapid development of computer and digital media technology, more and more visual artworks are created, preserved, and disseminated digitally [6]. People can access and enjoy a large number of high-quality digital art images on websites and mobile devices at any time. Faced with the ever-increasing mass of graphics and image data, its 
aesthetic evaluation cannot be completed completely by manual means [7]. If the computer can be used for their efficient automatic quantitative aesthetic evaluation, not only can save a lot of artwork management time and manpower consumption but also help ordinary users better understand and appreciate the artwork, at the same time, it is also of great significance to the advanced retrieval and style identification of artworks [8]. In addition, composition of some artistic graphics, no matter with simple shapes or ethnic art patterns with complex structures, must follow aesthetic principles. If we can use a computer to analyze their aesthetic characteristics and efficiently generate artistic patterns, we can quantitatively reveal the aesthetic law of artistic patterns, which is of great significance to modern pattern design and digital protection of national artistic patterns.

Their computing power to replace the human brain by computer processing analysis and visual perception system to an objective and quantitative understanding of visual art and the various properties of aesthetic cognition enable the computer to simulate the human visual system and aesthetic thinking to understand and deduce "beauty" of the calculable method and make feasible aesthetics in the related application of automatic decision [9]. This research establishes a bridge between computer and visual artworks, and its research purpose is to enable the computer to carry out quantitative analysis, calculation, and evaluation of the aesthetic feeling of graphics and images independently. For example, evaluate the aesthetic score of photos; judge the aesthetic style of paintings, so as to understand the aesthetic perception of users; and help find the most in line with the aesthetic needs of users [10]. At the same time, the computer can assist to generate a variety of visual aesthetic art forms and enhance the performance of visual art and creativity.

This paper presents a design method of visual communication effect evaluation of artworks based on machine learning. First, some characteristic variables are constructed to quantify the balance, contrast, and harmony in icon design criteria, and these three common design criteria are transformed into a mathematical expression. Then, the artificial evaluation scores are regressed to the calculated characteristic variables to obtain the statistical linear regression models corresponding to the three design criteria This paper selects the digital art form in the field of graphics and images and carries out the research of computational aesthetics in a new dimension.

\section{Related Work}

In recent years, more and more scholars have begun to study and explore computational aesthetics in various aspects [11]. The research direction related to this paper can be divided into two categories: the first is the quantitative aesthetic evaluation of graphic images, and the second is the modeling and drawing of traditional artistic patterns based on aesthetic rules [12]. However, most of the current work takes photographs and oil paintings as research objects, while there are few attempts to calculate the aesthetic evaluation of ink and wash paintings. Next, we analyze the research status and methods of computational aesthetics-related technologies from the following aspects.

2.1. Graphic Calculation Aesthetic. It has long had its own aesthetic standards for the construction of "beauty" in the fields of photographs, paintings, and graphic design. For example, the authenticity, clarity, exposure and depth of field in photos, color contrast, color harmony, and light and shadow performance in paintings can provide useful ideas for the aesthetic evaluation of artistic works [13]. However, the expression and description of these attributes only exist in the realm of art, and how to quantify them is a challenge. Scholars from all over the world have explored and studied computational aesthetic models and their applications from different perspectives. Most of the work is based on datadriven methods to quantify the aesthetic criteria and aesthetic common sense in artistic works through design or automatic learning of appropriate visual features and to carry out an aesthetic evaluation of artistic works [14]. In addition, with the development of bioinformatics, neuroscience, psychology, and other theories, experts and scholars try to use relevant theories to guide the study of the relationship between human vision and aesthetic perception of artworks.

2.2. Human Visual Aesthetic Perception. As one of the natural attributes of human perception, some researches deeply analyze the principles of the human aesthetic experience of visual artworks from the perspective of neuroaesthetics and psychology, trying to discover the visual characteristics that affect human aesthetic perception [15]. At the neuro aesthetic level, a functional magnetic resonance experiment was used in literature [16] to verify the difference of aesthetic stimulation in human brain regions when people appreciate artistic works. The results show that two distinct brain regions are active. The first region is responsible for receiving specific visual stimuli, such as color, shape, spatial position, and movement, which reflect the early processing of human vision. The second region is responsible for combining these visual stimuli and producing a perception of beauty or ugliness. Chatterjee A proposed the hierarchical sequence of visual processing in visual aesthetics, which can be divided into early image feature input, medium feature element combination, and late aesthetic perception experience [17]. Starting from the modularization of the human visual system, Peters deduced six aesthetic visual modules of the brain: color, form, movement, space, depth of field, and human body, and analyzed and explored each module based on the principles of cognitive neuroscience and visual art [18]. Raham extracted the features of images such as symmetrical space, sparsity, brightness, color, and composition level from the perspective of human visual perception and analyzed their relationship with neural coding and aesthetic perception [19].

In the field of psychology, researchers have found some factors in images that stimulate human aesthetic preference, such as color, object contour curvature, line orientation and 
spatial composition, perceptual fluency, and so on. Wallraven used eye tracker technology to study people's eye movements during appreciation and aesthetic evaluation of artistic works [20]. It proves that people's vision always focuses on the main area of the work when they appreciate it and find a visual perception model that simulates human aesthetic observation. Although the above work is not directly related to our research, it is very enlightening for us to extract important visual features and aesthetic norms related to human aesthetic perception.

\subsection{Graphic Image Calculation Aesthetic Evaluation Method.} In recent years, the research on computational aesthetics of graphic design has achieved phased results. In terms of computational aesthetic evaluation of web pages, Zheng quantified the symmetry, balance, and equilibrium of web page layout structure by extracting statistical features of lowlevel images [21]. Reinecke extended previous work and proposed the first perception model of web page visual complexity and chroma, which was used as a predictive variable to evaluate users' aesthetic impression of web page design [22]. As the aesthetic factors in web pages include visual chroma, the relationship and order between modeling elements, highlighting the main elements, and so on, and these factors do not exist in the icon design criteria, the visual law of icon design is also very different from the website. Therefore, the existing computational aesthetic evaluation framework of web pages is not completely applicable to the computational aesthetic evaluation of artworks.

There is also some research work to establish the relationship between computer vision and the aesthetic properties of graphic design. Liao studied key visual elements in icon design by extracting visual features, including complexity, balance, and repeatability [23]. However, they only carried out statistical analysis on the distribution of calculated characteristic variable values and did not use manual evaluation to verify the validity of the method. Furthermore, these visual elements do not correlate with the aesthetic perception of the artworks. Wang discovered the color rules in corporate ICONS by calculating the number of main hues and the correlation between hue, saturation, and lightness [24]. Saleh used low-level visual features to model the similarity of information graph [25]. They evaluated different visual features and learning algorithms and found that the combination of the color histogram and gradient histogram features was the most effective way to describe the stylistic characteristics of the information graph. The above work has great enlightenment for us in extracting important visual features and aesthetic criteria related to human and artistic works' aesthetic perception.

\section{Theory and Method}

3.1. Overall Framework of the Model. Figure 1 shows our computing aesthetic evaluation framework. For each image (yellow box) in the artworks data set, we follow the following four steps. First, we detect the area and corresponding edge (the green box) of each image. Second, we construct a set of characteristic variables and use them to quantitatively assess each icon's balance, contrast, and perception of harmony (blue box). To verify the validity of the computational assessment method, we performed manual assessments of balance, contrast, harmony, and aesthetic perception (pink box). Third, the corresponding linear regression model (orange box) is obtained by regression of the balance, contrast, and harmony scores of manual evaluation to these characteristic variables respectively. Finally, the aesthetic model (red box) was obtained by regression of the balance, contrast, and harmony scores of the artificial evaluation of aesthetic score to the model evaluation.

We use $G$ to represent an icon that contains multiple areas organized together by design principles such as balance, contrast, harmony, variation, and rhythm to form complete artworks. We call each region in $G$ a visual element. Here, we use the region props function provided in MATLAB to detect all regions in $G$.

\subsection{Quantitative Calculation of Criteria for Works of Art.} This section will introduce the quantitative evaluation methods of icon balance, contrast, and harmony in detail. For each dimension, we will introduce the corresponding characteristic variable construction and calculation methods.

Balance refers to the balanced distribution of visual weight in a work of art to achieve a state of equilibrium to create a sense of calm. The most common work of art is leftright balance, in which the works with left-right symmetrical balance show a stable feeling. Some works of art with dynamic effects are designed as asymmetric balance. Therefore, measuring the left-right balance of artwork $G$ is more challenging than simply detecting its left-right symmetry. Here, we calculate the left and right equilibrium degree of $G$ by constructing the weight difference curve.

The length and width of $G$ are denoted by $H$ and $W$, respectively. The vertical axis of symmetry of $G$ is indicated by the irrigation. Considering the space left by the white area for the black area, the two together make up the shape of the image, as shown in Figure 2.

If the black areas to the left and right of the ribbon are equally weighted, then $G$ is considered to be left and right balanced. Therefore, we scan $G$ line by line from top to bottom. On each scan line, we first select the pixels falling into the black area from left to right $P_{i, j}$. We define the weight sets represented by the black area to the left and right of $X_{v}$ on the $i$-scan line, respectively, as $d l_{i}$ and $d r_{i}$. And then we calculate the absolute distance between $P_{i, j}$ and $X_{v}$. If $P_{i, j}$ is to the left of $X_{v}$, we put it in $d l_{i}$, and if it is to the right, we put it in $d r_{i}$. The weight difference between $d l_{i}$ and $d r_{i}$ on $i$-th scan line is defined as follows:

$$
D_{i}=\frac{1}{M l_{i}} \sum_{j=1}^{M l_{i}} d l_{i, j}-\frac{1}{M r_{i}} \sum_{k=1}^{M r_{i}} d r_{i, k},
$$

where $M l_{i}$ and $M r_{i}$ refer to the number of distance elements contained in $d l_{i}$ and $d r_{i}$, respectively; and $d l_{i, j}$ and $d l_{i}$ refer 


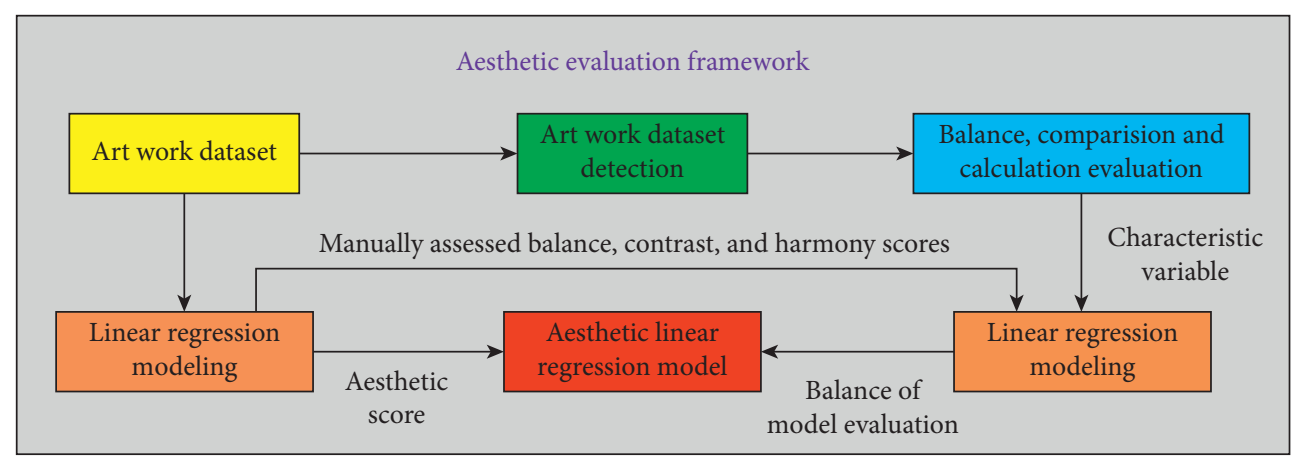

FIgURE 1: Computational aesthetic evaluation frame for works of art.

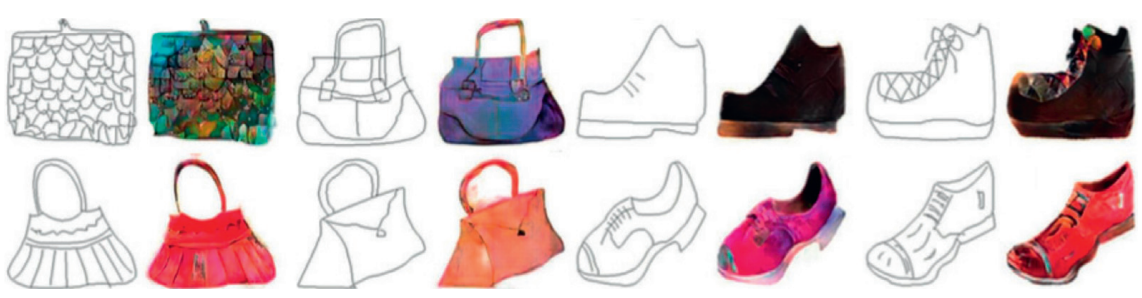

Figure 2: The two together form a schematic diagram of the shape.

to the $j$-th distance element; and $d r_{i, k}$ refer to the $k$-th distance element in $d r_{i}$. We calculate the $D_{i}$ of all scans to obtain the weight difference curve. If $D_{i}=0$ for all $i \in[1, H]$, then $G$ is left and right symmetric equilibrium. Otherwise, $G$ from top to bottom must contain some sloping parts with different zigzag variations. Here, the square root of the absolute value of the area difference between curve $D$ and the area above and below the horizontal axis is expressed as $S$, which is used to measure the left and right balance of $G$. The smaller $S$ is, the more balanced $G$ is.

Contrast designations, colors, textures, shapes, and other elements to create visual excitement and add interest to the design work. Since images can be multiple colors, single colors, or metallic colors. Here, we transform all the collected images into black and white to focus on the contrast in shape. Generally, shapes with sharp corners attract more attention than smooth curves; they further suggest that people are born with a deep preference for curves rather than sharp corners. In the design, the corners are used to attract visual attention, while the curved parts are used to create a positive mood and aesthetic impression. So, here, we propose rigid and flexible contrast to evaluate shape contrast in images. Attneave found in an experiment that the information was mainly concentrated on the contour of the shape and further concentrated on some boundary points on the contour where the direction changed rapidly [26]. Inspired by these conclusions, we adopt Shannon information theory to calculate rigid and flexible pairs; the calculation process is described in detail as follows.

In the discrete case, when the curve is sampled uniformly, the curvature is proportional to the turning angle $\alpha$, which changes tangentially from one point to another along the shape profile. We assume that it follows the von Mises distribution centered on the straight line $\alpha=0$; the mathematical expression is as follows:

$$
p(\alpha)=A \exp [b \cos (\alpha)]
$$

where $b$ is the parameter used to adjust the diffusion degree of the distribution and $A$ is the normalized constant term. If $\alpha$ is positive, it means clockwise rotation, and a negative value means counterclockwise rotation, as shown in Figure 3.

We define the set of corner points and sampling points in $G$ as $\varphi_{c}$ and $\varphi_{s}$. For each visual element $E_{k}$ in $G$, we first use a CSS corner detection algorithm to detect corners and put them into the collection. These corners correspond to sharp vertices in $B P_{k}$ and divide $B P_{k}$ into several line segments, including arcs or lines. Then, between the two corners of each curve, we take samples at every five edge points starting from one corner along the $B P_{k}$ and call these sample points breakpoints. Finally, we take corner points and breakpoints as control points and use the arc length parameterization method to uniformly sample the curve. We continue this process until all line segments in $B P_{k}$ are sampled.

Shannon defined the amount of information as the negative value of the logarithmic probability distribution. Therefore, the amount of information of a vertex with steering angle $\alpha$ is calculated as follows:

$$
I_{\alpha}=-\log _{2} p(\alpha)
$$

where the corresponding information of rigid point (corner point) and soft point (sampling point) is, respectively, calculated as follows: 


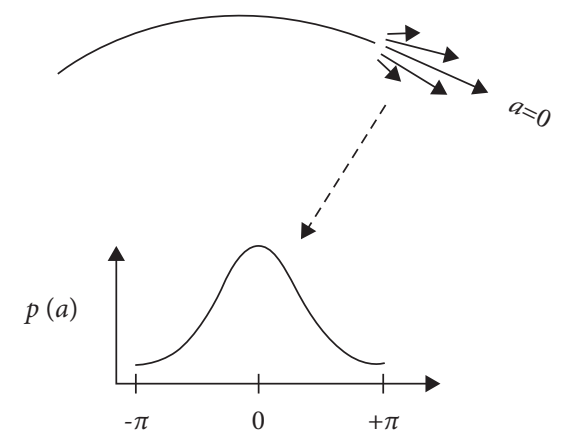

FIGURE 3: Schematic diagram showing distribution centered on the straight line.

$$
\begin{aligned}
& I_{h}=-\sum_{\operatorname{vertex}(\alpha) \in \Psi_{c}} \log _{2} p(\alpha) \\
& I_{s}=-\sum_{\operatorname{vertex}(\alpha) \in \Psi_{s}} \log _{2} p(\alpha)
\end{aligned} .
$$

Finally, the rigidity and flexibility of $G$ compared with $C_{h s}$ are calculated as follows:

$$
C_{h s}=\frac{I_{s}}{I_{h}+I_{s}},
$$

where $C_{h s}$ is between 0 and 1 . When $C_{h s}$ closer to 1 , the softer the images $G$ becomes. The closer this value approaches 0 , the stiffer images $G$ becomes. In the beginning, we used equation (5) to calculate the rigid-flexible contrast of images. However, for some icons surrounded by one or more closed outer boundaries, the calculation results are very different from the manual evaluation results. We speculate that the outer and inner boundaries contribute to the overall rigid and flexible contrast of these icons, respectively. Therefore, we use $C_{\text {out }}$ and $C_{\text {in }}$ represent the rigid and flexible contrast of the outer boundary (if present in $G$ ) and the inner boundary, respectively, and are calculated as follows:

$$
\begin{gathered}
C_{\text {out }}=\frac{I_{\text {sout }}}{I_{\text {hout }}+I_{\text {sout }}}, \\
C_{\text {in }}=\frac{I_{\text {sin }}}{I_{\text {hin }}+I_{\text {sin }}},
\end{gathered}
$$

where $I_{\text {hout }}$ and $I_{\text {sout }}$ represent the total amount of information corresponding to the rigid and soft points on the outer boundary, respectively, and $I_{\text {hin }}$ and $I_{\text {sin }}$ represent the information amount corresponding to the rigid point and the soft point on the inner boundary, respectively. Harmony is a design criterion that measures how the visual elements of a work of art fit together. It provides the cohesion that makes the work of art a perfect whole. For visual elements in icon design, harmony means that the shape of a visual element should match that of its neighboring visual elements. Among these visual elements, the areas between some adjacent holes make a great contribution to the overall harmony of the images. In this section, we define normal tracking distance to calculate the coincidence degree between different adjacent visual elements in the icon, as detailed below.
Previously, we have detected the boundary $B_{k}$ of each visual element rat in $G$ and its corresponding feature point set $F P_{k}$ most. We connect every two feature points with line segments so that $B_{k}$ can be approximated as a polygon composed of a series of line segments. Then, for the midpoint of each line segment, we select the boundary point closest to that position at night. We represent this point as SP and start from this point along the outer normal direction of the line segment.

3.3. SVR Algorithm Theory. SVM is generally divided into support vector classification machine and support vector regression machine. SVC is used to deal with classification problems, which can deal with both two types of classification problems and multiple types of classification problems; SVR is used to deal with functional regression [27]. SVR has been successfully applied to system identification and nonlinear system prediction. The basic idea of support vector function regression is: for a given training sample point $\{(x, y), \ldots,(x, y)\} \subset X \times R$, a function $f(x)$ is regressed through SVR training so that the difference between the output value of each sample calculated by the function and the target value corresponding to the input sample does not exceed the error, and the regressed function is as smooth as possible [28].

In the case of linear regression, suppose that the form of the function is: $f(x)=(w \cdot x)+b$, where $(\cdot)$ represents the dot product in $x$. To make the regressed function $f(x)$ as smooth as possible, it is necessary to find a $w$ as small as possible. Therefore, the above problem can be described as a convex optimization problem:

$$
\begin{aligned}
& \min \frac{1}{2}\|w\|^{2}, \\
& \text { s.t. }\left\{\begin{array}{l}
\left(\left(w \cdot x_{i}\right)+b\right)-y_{i} \leq \varepsilon, \\
y_{i}-\left(\left(w \cdot x_{i}\right)+b\right) \leq \varepsilon, \quad i=1,2, \ldots, l .
\end{array}\right.
\end{aligned}
$$

In practice, some small errors are often ignored, so the relaxation factor is introduced $\xi_{i}$ and $\xi_{i}^{*}$, so the above formula is usually written as follows:

$$
\begin{aligned}
& \min \frac{1}{2}\|w\|^{2}+C \sum_{i=1}^{t}\left(\xi_{i}^{*}+\xi_{i}\right), \\
& \text { s.t. }\left\{\begin{array}{l}
\left(\left(w \cdot x_{i}\right)+b\right)-y_{i} \leq \varepsilon+\xi_{i} \\
y_{i}-\left(\left(w \cdot x_{i}\right)+b\right) \leq \varepsilon+\xi_{i}^{*} \quad i=1,2, \ldots, l . \\
\xi_{i}, \xi_{i}^{*} \geq 0
\end{array}\right.
\end{aligned}
$$

The constant $C>0$ is the balance factor, which determines the balance between the smoothness of the function and the number of sample points whose value error exceeds. The above problem is a convex quadratic optimization problem. In order to obtain its solution, the Lagrange function is introduced as follows: 


$$
\begin{aligned}
L= & \frac{1}{2}\|w\|^{2}+C \sum_{i=1}^{l}\left(\xi_{i}^{*}+\xi_{i}\right)-\sum_{i=1}^{l} \alpha_{i}\left(y_{i}-\left(w \cdot x_{i}\right)-b+\varepsilon+\xi_{i}\right) \\
& -\sum_{i=1}^{1} \alpha_{i}^{*}\left(\left(w \cdot x_{i}\right)+b-y_{i}+\varepsilon+\xi_{i}^{*}\right)-\sum_{i=1}^{l}\left(\gamma_{i}^{*} \xi_{i}^{*}+\gamma_{i} \xi_{i}\right),
\end{aligned}
$$

where $\alpha_{i}, \alpha_{i}^{*}, \gamma_{i}, \gamma_{i}^{*} \geq 0, \quad i=1,2, \cdots, l$. At the extreme point of function $L$, the partial derivative relative to variable $\left(w, b, \xi_{i}, \xi_{1}^{*}\right)$ is equal to zero, that is:

$$
\begin{gathered}
\frac{\partial}{\partial w} L=w-\sum_{i=1}^{1}\left(\alpha_{i}-\alpha_{i}^{*}\right) x_{i}=0 \\
\frac{\partial}{\partial b} L=\sum_{i=1}^{1}\left(\alpha_{i}-\alpha_{i}^{*}\right)=0 \\
\frac{\partial}{\partial \xi^{(0)}} L=C-\alpha_{i}^{(\theta)}-\gamma_{i}=0 .
\end{gathered}
$$

Substituting the above three formulas into function $L$ produces the corresponding dual optimization problem as follows:

$$
\begin{aligned}
\operatorname{Max} W\left(\alpha, \alpha^{*}\right)= & \frac{1}{2} \sum_{i=1}^{1} \sum_{J=1}^{1}\left(\alpha_{i}^{*}-\alpha_{i}\right)\left(\alpha_{j}^{*}-\alpha_{j}\right)\left(x_{i} \cdot x_{j}\right) \\
& +\varepsilon \sum_{i=1}^{l}\left(\alpha_{i}^{*}+\alpha_{t}\right)-\sum_{i=1}^{l} y_{i}\left(\alpha_{i}^{*}-\alpha_{i}\right) .
\end{aligned}
$$

The constraints are as follows:

$$
\sum_{i=1}^{1}\left(\alpha_{i}^{*}-\alpha_{i}\right)=0 \alpha_{i}, \alpha_{i}^{*} \in[0, C] ; \quad i=1,2, \ldots, l .
$$

How to deal with the nonlinear situation in support vector function regression SVR? The basic idea of the support vector machine is to nonlinearly transform the input sample space to another feature space and construct the regression function in this feature function space. The specific methods are as follows: first, the input samples are mapped to a feature space through a function, in which the samples are linear, so the training algorithm in linear regression described above can be applied. Then, the kernel function is introduced to replace the point product between samples in the feature space. The mathematical expression is as follows:

$$
\begin{aligned}
\operatorname{Max} W\left(\alpha, \alpha^{*}\right)= & \frac{1}{2}\left(\alpha_{i}^{*}-\alpha_{i}\right)\left(\alpha_{j}^{*}-\alpha_{j}\right) K\left(x_{i} \cdot x_{j}\right) \\
& +\varepsilon \sum_{i=1}^{t}\left(\alpha_{i}^{*}+\alpha_{i}\right)-\sum_{i=1}^{1} y_{i}\left(\alpha_{i}^{*}-\alpha_{i}\right) .
\end{aligned}
$$

The kernel function used in the nonlinear support vector regression function is the same as that used in nonlinear support vector classification. The method of calculating deviation $b$ is the same as that of kernel linear regression. It can be seen from the above analysis that in the case of nonlinear regression, the vector $W$ is no longer given directly like linear regression. In addition, nonlinear regression is not in the input space but in the feature space to find the smoothest regression function. The main reason for preselecting effective candidate support vectors is that the support vectors are usually located at the class boundary. Compared with other samples of this class, they are far from the center of this class and close to the center of dissimilarity. Therefore, select the samples whose mutual center distance (the distance between samples and dissimilarity centers) is less than the center distance between the two types of samples as effective candidate support vectors, as shown in Figure 4 .

\section{Experiments and Results}

4.1. Experimental Results of Regression Modeling. The experimental environment of this article is as follows: the hardware environment is a Linux system, NVIDIA GTX 2080Ti; the software environment is Python3.5, sklearn0.21.1, and other toolkits. Similar to previous work, we have used the public data set and the same data partitioning method [24-26]. In this section, we use the linear regression model based on machine learning to analyze the relationship between the manually evaluated balance, rigidflexible contrast, harmony score, and various feature variables extracted from geometric attributes. On this basis, we further analyze the relationship between the aesthetic score of artificial evaluation and the balance, rigidity-flexible contrast, and harmony score of regression model evaluation. We used statistical analysis software SPSS and statistical analysis package based on regression analysis to perform regression modeling of balance, rigidity-flexible contrast, harmony, and aesthetic feeling. Regression analysis enables us to verify the validity of regression models by the degree of fit, to verify statistical significance by the F-test of overall model fit, and to select significant predictive variables using the $T$-test. In order to compare the relative importance of each variable more easily, we normalized each characteristic variable using min linear transformation. Then, for the three dimensions of balance, rigid-flexible contrast, and harmony, the Monte Carlo cross-verification method was adopted in model training.

The statistical results of the regression model for balance perception are shown in Table $1 . S$ is the square root of the absolute value of the area enclosed by the weight difference curve $D$ and the horizontal axis. Table 1 shows that our model can explain $94.1 \%$ of the variation in human balance perception and is statistically significant. Since the $p$ value corresponding to $S$ is less than 0.05 , it makes a significant contribution to our model. It shows that the weight difference curve $D$ can reasonably describe the left and right visual weight distribution in the icon; $S$ can effectively describe the balance perception of images.

Statistical results of the regression model for rigidflexible contrast perception are shown in Table 2; $C_{\text {out }}$ and $C_{\text {in }}$ describe the rigid and flexible contrast of the inner and outer boundaries of the icon, respectively. Table 2 indicates that our model can explain $93 \%$ of the variation in human rigid-flexible contrast perception and is statistically 

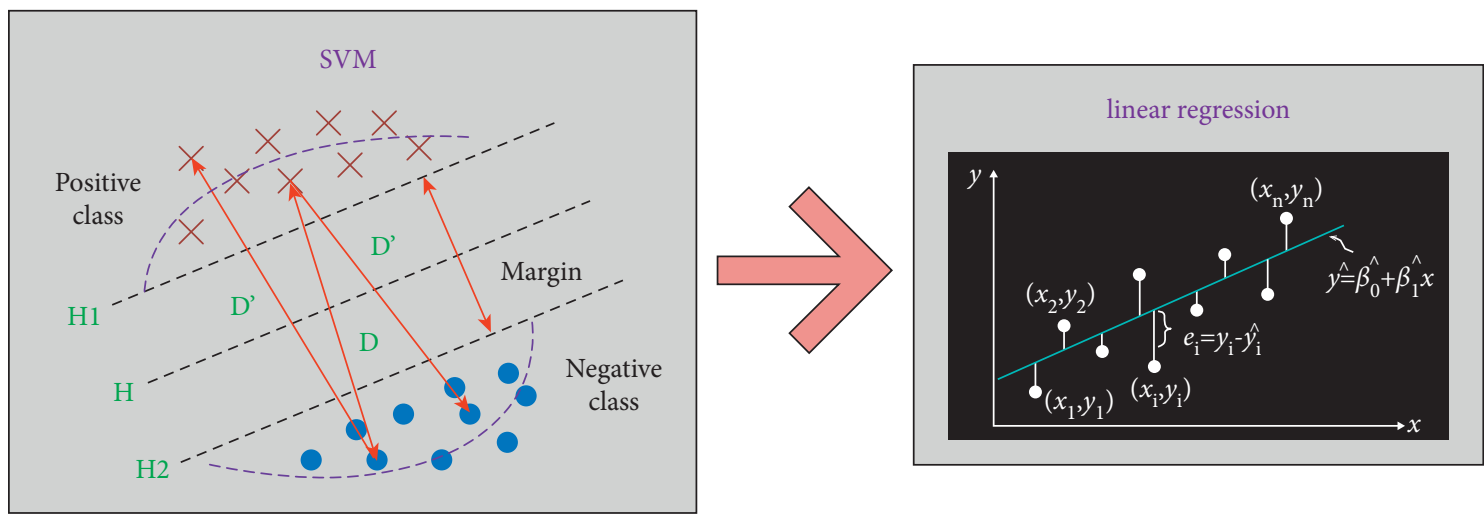

FIGURE 4: Schematic diagram of SVR algorithm.

TABLE 1: The balance perception regression model is the statistical result.

\begin{tabular}{lccccc}
\hline$R^{2}=0.943$ & \multicolumn{2}{c}{ Adj. $R^{2}=0.941$} & Std.Er $=0.267$ & $F=470.736$ & \multicolumn{1}{c}{ Sig $=0.00$} \\
\hline Var & Coef & Er & Coef & Sig. \\
$S$ & 0.997 & 0.051 & 0.971 & 13.908 & 0.000 \\
Const & -0.155 & 0.42 & - & -3.548 & 0.003 \\
\hline
\end{tabular}

TABLe 2: Statistical results of rigid-flexible contrast perception regression model.

\begin{tabular}{llcccc}
\hline$R^{2}=0.935$ & \multicolumn{2}{c}{ Adj. $R^{2}=0.930$} & Std.Er $=0.257$ & $F=226.206$ & \multicolumn{2}{c}{ Sig $=0.00$} \\
\hline Var & Coef & Er & Coef & Sig. \\
$C_{\text {out }}$ & 0.299 & 0.038 & 0.451 & 7.914 & 0.000 \\
$C_{\text {in }}$ & 0.527 & 0.047 & 0.648 & 10.862 & 0.003 \\
Const & 0.388 & 0.098 & - & 3.968 & 0.000 \\
\hline
\end{tabular}

significant. Because of $C_{\text {out }}$ and $C_{\text {in }}$, the corresponding $p$ values are less than 0.05 , so they contribute significantly to our model.

The statistical results of the regression model of harmony perception are shown in Table 3. Fit represents the average Fit between all adjacent visual elements in the image. The model explained $86.3 \%$ of the variation in human perception of harmony and was statistically significant. Since the $p$-value corresponding to Fit is less than 0.05 , it makes a significant contribution to our model. This shows that the normal tracking distance we calculated can properly describe the coincidence between adjacent visual elements in the icon, and fit can effectively describe the harmony perception of the image.

We have obtained regression models $C_{b}, C_{c}$, and $C_{h}$ of balance perception, rigid-soft contrast perception, and harmony perception, respectively. On this basis, we take these three models as predictive variables to construct a regression model of human aesthetic perception. The overall statistical results of the regression model are shown in $\mathrm{Ta}$ ble 4 . The model explained $98.3 \%$ of the variation in human aesthetic perception and was statistically significant. Since $p$-values corresponding to $C_{b}, C_{c}$, and $C_{h}$ are less than 0.05 , they have a significant contribution to our model.

In this section, we will further analyze and evaluate the regression model performance of balance, rigid-flexible contrast, harmony, and aesthetic perception in the previous section. We have got the regression model of balance, rigid and flexible contrast, harmony, and beauty. Figure 5 shows the comparison of artificial evaluation scores (blue curve) and regression model evaluation scores (red curve) of 60 images in each dimension. As can be seen from the figure, the evaluation result of our regression model approximates the artificial evaluation result.

Figure 5 shows the average RPE statistical results of the balance, rigid-flexible contrast, harmony, and aesthetic regression model under eight runs, in which the green curve represents the training set and the red curve represents the test. In order to better evaluate the prediction performance of the regression model, we show the average Pearson correlation coefficient and corresponding bilateral significance of each model training set and test set under eight runs, as well as the average mean square error between the model evaluation results and the manual evaluation results, which is used to predict the average prediction performance of the regression model under different training sets and test sets. All these prove that our regression model has high prediction performance, and the explanatory variables of the regression model have high prediction ability in icon computing aesthetic evaluation. We show some icon comparisons in which the evaluation scores on four aesthetic attributes of balance, rigid and soft contrast, harmony, and beauty show opposite directions, as shown in Figure 6. 
TABLE 3: Statistical results of a harmonious perception regression model.

\begin{tabular}{lccccc}
\hline$R^{2}=0.866$ & \multicolumn{2}{c}{ Adj. $R^{2}=0.863$} & Std.Er $=0.301$ & $F=176.968$ & Sig $=0.00$ \\
\hline Var & Coef & Er & Coef & Sig. \\
$S$ & 0.824 & 0.055 & 0.945 & 14.266 & 0.000 \\
Const & -0.437 & 0.148 & - & 2.872 & 0.006 \\
\hline
\end{tabular}

TABLE 4: Statistical results of an aesthetic perception regression model.

\begin{tabular}{llcccc}
\hline$R^{2}=0.985$ & \multicolumn{2}{c}{ Adj. $R^{2}=0.983$} & Std.Er $=0.321$ & $F=998.206$ & Sig $=0.00$ \\
\hline Var & Coef & Er & Coef & Sig. \\
$C_{\text {out }}$ & 0.711 & 0.045 & 0.788 & 16.102 & 0.000 \\
$C_{\text {in }}$ & 0.172 & 0.055 & 0.146 & 5.807 & 0.000 \\
Const & 0.086 & 0.046 & 0.073 & 2.903 & 0.000 \\
\hline
\end{tabular}

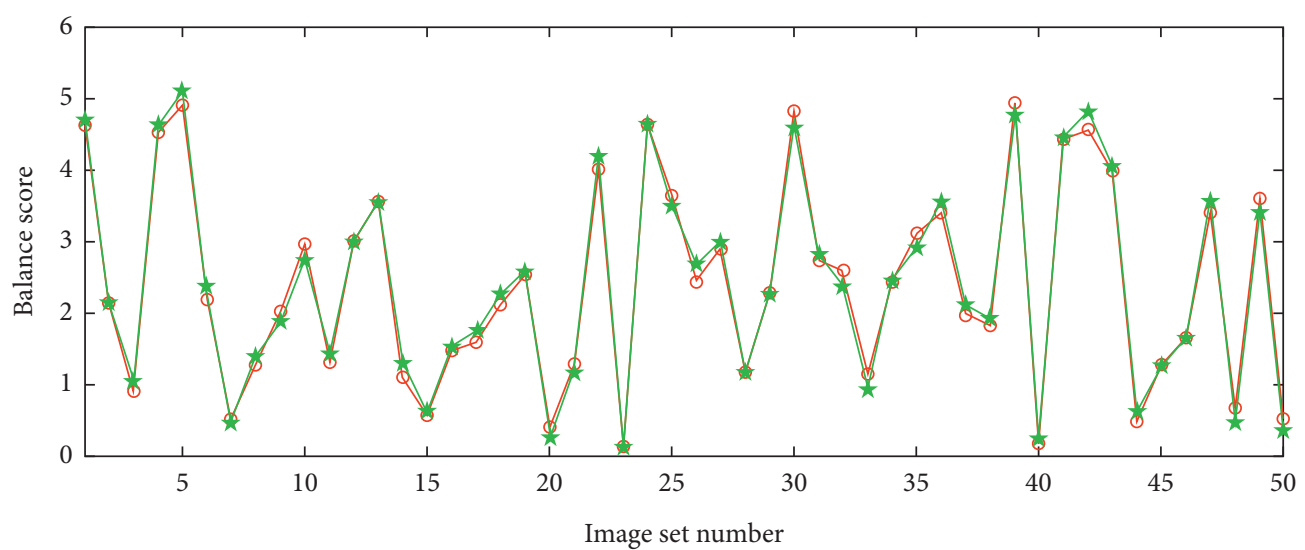

- - Manual evaluation

$\star$ regression model

FIgURe 5: Comparison between the regression model and manual evaluation results.

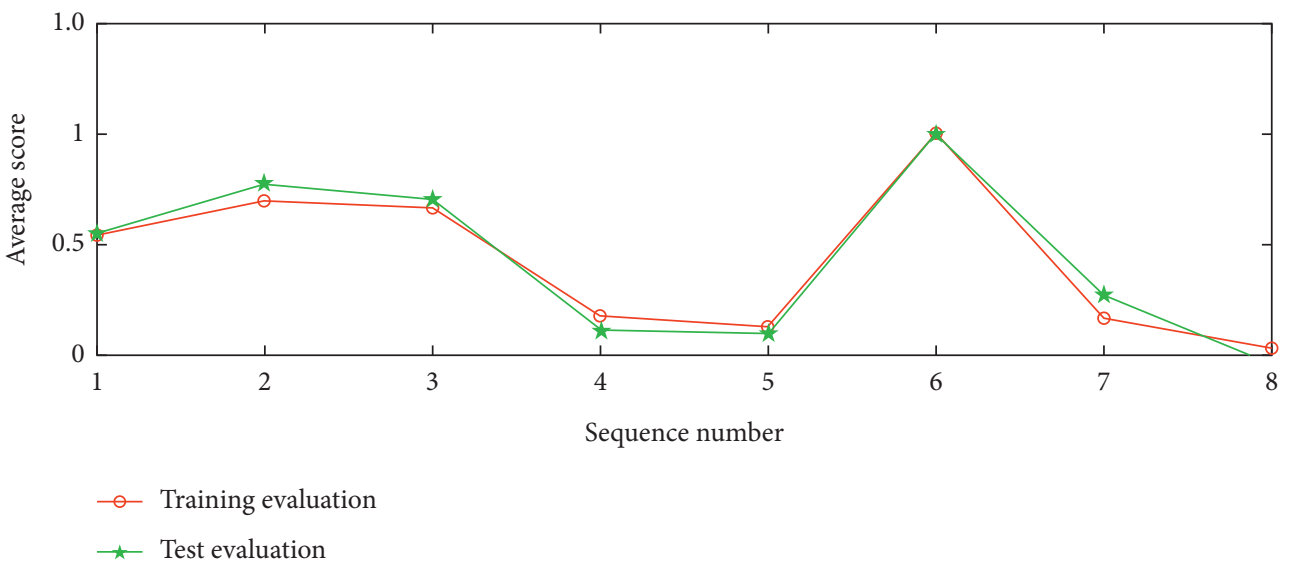

FIGURE 6: Schematic diagram of performance results.

The values in the figure show that our regression model can well describe and predict each aesthetic attribute and is consistent with human aesthetic perception.
4.2. Experimental Results of Computational Aesthetic Evaluation. In order to make the characteristic variables insensitive to proportional changes, we use the corresponding 
TABle 5: Performance statistical results of different models under 10 runs.

\begin{tabular}{lcccc}
\hline & $M_{c}$ & $M_{t}$ & $M_{p}$ & $M_{\alpha}$ \\
\hline Average & $0.864 /$ & $0.834 /$ & $0.904 /$ & $0.822 /$ \\
$R_{p} /$ Sig & 0.000 & 0.000 & 0.000 & 0.000 \\
SD $R_{p} /$ Sig & $0.023 /$ & $0.047 /$ & $0.015 /$ & $0.024 /$ \\
Average MSE & 0.000 & 0.000 & 0.000 & 0.000 \\
SD MSE & 0.649 & 0.887 & 0.478 & 0.945 \\
& 0.032 & 0.022 & 0.027 & 0.042 \\
\hline
\end{tabular}
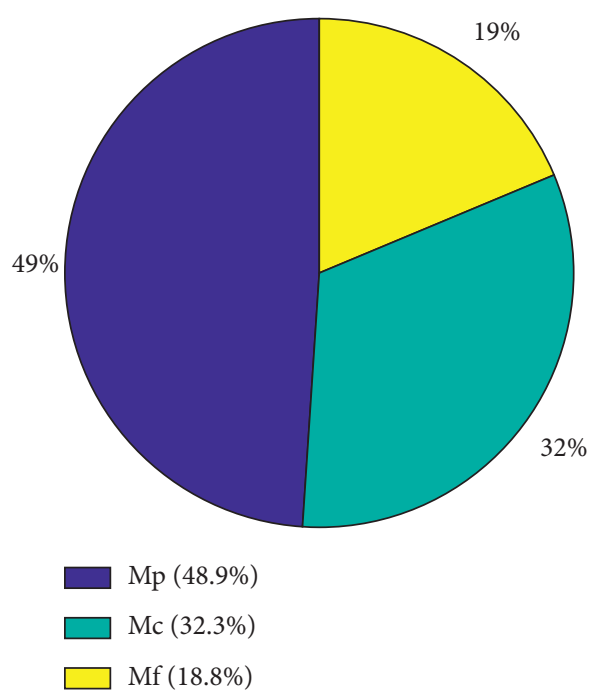

FIGURE 7: Proportion of each model category and characteristic variable in the aesthetic model.

weighted average and standard deviation to standardize each characteristic variable. In the experiment, we use the statistical and machine learning toolbox in MATLAB to establish the SVR model. In order to avoid overfitting, we use nested fivefold cross-validation to train color, texture, and composition-aware SVR models. Table 5 shows the performance statistical results of different models under 10 runs.

The outer fivefold cross-validation loop randomly divides the data set into five equal subsets to estimate the generalization prediction performance of the training model. In the five subsets, each subset was selected as the test set in turn, and the remaining four subsets were selected as the training set. After model training, the test set is used to evaluate model performance. We show comparisons of some ink paintings that show opposite directions in predicting the assessment scores of aesthetic perception, as shown in Figure 7. The aesthetic score of the top line works is higher than that of the bottom line works. The values in the figure show that our aesthetic model can well describe and predict the aesthetic attributes of the ink and wash paintings, which is consistent with human aesthetic perception.

\section{Conclusion}

This paper deeply studies the related contents of image computing aesthetic evaluation and puts forward a design of visual communication effect evaluation method of artworks based on machine learning. First, this paper proposes an icon computing aesthetic evaluation framework based on the design criteria perception model. It first constructs some characteristic variables to quantify the balance, contrast, and harmony in the icon design criteria, then regresses the manual evaluation score to the calculated characteristic variables to obtain the statistical linear regression model corresponding to the three design criteria, and finally regresses the manual evaluation aesthetic score to the balance, contrast, and harmony scores of the model evaluation to obtain the aesthetic model. The experimental results show that our regression model can predict human judgments on balance, contrast, harmony, and aesthetic perception and has a highly significant correlation. Our work provides a reference framework based on machine learning for the quantitative aesthetic evaluation of graphic design, establishes the relationship between human aesthetic perception and computable design criteria extracted from graphic design.

Our current research on icon aesthetics is limited to shape aesthetics, while color plays a certain role in icon calculation aesthetic evaluation. In the future, we will incorporate color into the icon calculation aesthetic evaluation. In addition, the images studied in this paper are all from famous brand enterprises, and most of them are professionally designed. Therefore, our model may have poor expansibility for random or poorly designed images, so we need to add some randomly designed images in the training set to improve our model.

The computational aesthetic evaluation framework establishes the relationship between human aesthetic perception and the extracted computable visual features. First, it extracts some feature variables related to color, texture, and composition in the image and then regresses the artificial evaluation scores to the calculated feature variables to obtain the SVR model corresponding to the three aesthetic attributes. Finally, the artificial aesthetic score is regressed to the color, texture, and composition scores evaluated by the model, so as to obtain the aesthetic model.

Finally, the artistic patterns are dense, exquisite, and changeable. Each layer from the inner layer to the outer layer contains many types of decorative patterns. In future work, we will study the computer generation methods of other decorative patterns in the artistic patterns and integrate the generation model of decorative patterns into our user interaction system to generate more complex artistic patterns. In addition, the art troupe has bright colors and a distinctive style. Quantifying the aesthetic law contained in it is also a direction worthy of exploration.

\section{Data Availability}

The data sets used during the current study are available from the corresponding author on reasonable request.

\section{Conflicts of Interest}

The authors declare that there are no conflicts of interest. 


\section{Acknowledgments}

This work is supported by the following projects: (1) the design of intelligent classification garbage can for public and household. The Science and Technology project was supported by the Science and Technology Department of Anyang, established in 2018 (project number: 20262). (2) Color vision accessibility products design based on WCAG2.1 and Mathematica. The Foundation for Fostering the Scientific Research of Anyang Institute of Technology in 2020, established in 2020 (project number: YPY2020018). (3) The application of virtual exhibition in the dissemination of campus culture-take Anyang Institute of Technology as an example, Henan Province College Student Innovation and Entrepreneurship Training Program in 2020, established in 2020 (project number: S202011330021).

\section{References}

[1] H. Hagtvedt, V. M. Patrick, and R. Hagtvedt, "The perception and evaluation of visual art," Empirical Studies of the Arts, vol. 26, no. 2, pp. 197-218, 2008.

[2] L.-Y. Zhu, The Great Aesthetic Dictionary, Shanghai Dictionary Publishing House, Shanghai, China, 2010.

[3] A. Chatterjee, "Neuroaesthetics: a coming of age story," Journal of Cognitive Neuroscience, vol. 23, no. 1, pp. 53-62, 2011.

[4] J. Zhang, J. Yu, K. Zhang, X. S. Zheng, and J. Zhang, "Computational aesthetic evaluation of logos," ACM Transactions on Applied Perception, vol. 14, no. 3, pp. 1-21, 2017.

[5] L. J. Roosen, C. A. Klöckner, and J. K. Swim, "Visual art as a way to communicate climate change: a psychological perspective on climate change-related art," World Art, vol. 8, no. 1, pp. 85-110, 2018.

[6] S. Khan, H. Rahmani, S. A. A. Shah, and M. Bennamoun, "A guide to convolutional neural networks for computer vision," Synthesis Lectures on Computer Vision, vol. 8, no. 1, pp. 1-207, 2018.

[7] M. Wevers and T. Smits, "The visual digital turn: using neural networks to study historical images," Digital Scholarship in the Humanities, vol. 35, no. 1, pp. 194-207, 2020.

[8] W. R. Williams, "Attending to the visual aspects of visual storytelling: using art and design concepts to interpret and compose narratives with images," Journal of Visual Literacy, vol. 38, no. 1-2, pp. 66-82, 2019.

[9] B. F. Spencer, V. Hoskere, and Y. Narazaki, "Advances in computer vision-based civil infrastructure inspection and monitoring," Engineering, vol. 5, no. 2, pp. 199-222, 2019.

[10] N. G. Ikhtiyorovna, "Aesthetic education and methods of aesthetic development in children with disabilities," Middle European Scientific Bulletin, vol. 3, pp. 4-6, 2020.

[11] L. Yue, G. Chao, L. Yi-Lun, Z. Fan, and W. Feiyue, "Computational aesthetics of fine art paintings: the state of the art and outlook," Acta Automatica Sinica, vol. 46, no. 11, pp. 2239-2259, 2020.

[12] V. Kitsopoulou, Analysing Formal Visual Elements of Corporate Logotypes Using Computational Aesthetics, University of East Anglia, Norwich, UK, 2018.

[13] J. Pei, K. Zhong, J. Li, and X. Wang, "ECNN: evaluating a cluster-neural network model for city innovation capability," Neural Computing \& Applications, 2021.

[14] C. Wang and X. Ren, "An entropy-based approach for computing the aesthetics of interfaces," in Proceedings of the
2018 ACM Companion International Conference on Interactive Surfaces and Spaces, pp. 57-61, Tokyo, Japan, November 2018.

[15] H. Jokeit and D. Blochwitz, "Neuro-aesthetics and the iconography in photography," PsyCh Journal, vol. 9, no. 4, pp. 444-457, 2020.

[16] H. Kawabata and S. Zeki, "Neural correlates of beauty," Journal of Neurophysiology, vol. 91, no. 4, pp. 1699-1705, 2004.

[17] A. Chatterjee, "Prospects for a cognitive neuroscience of visual," Bulletin of Psychology and the Arts, vol. 4, no. 2, pp. 56-60, 2004.

[18] G. Peters, "Aesthetic primitives of images for visualization," in Proceedings of the 11th International Conference on Information Visualization, pp. 316-325, IEEE, Zurich, Switzerland, July 2007.

[19] D. J. Graham and C. Redies, "Statistical regularities in art: relations with visual coding and perception," Vision Research, vol. 50, no. 16, pp. 1503-1509, 2010.

[20] C. Wallraven, D. Cunningham, and J. Rigau, "Aesthetic appraisal of art-from eye movements to computers," in Proceedings of the International Symposium on Computational Aesthetics in Graphics, Visualization and Imaging, pp. 137144, Euro-Graphics Association, Switzerland, May 2009.

[21] X. Zheng, I. Chakraborty, J. Lin, and R. Rauschenberger, "Correlating low-level image statistics with user's rapid aesthetic and affective judgments of web pages," in Proceedings of the SIGCHI Conference on Human Factors in Computing Systems, pp. 1-10, ACM, New York, NY, USA, April 2009.

[22] K. Reinecke, T. Yeh, L. Miratrix, and R. Mardiko, "Predicting users first impressions of web- site aesthetics with a quantification of perceived visua complexity and colorfulness," in Proceedings of the Conference on Human Factors in Computing Systems, pp. 2049-2058, ACM, New York, NY, USA, April 2013.

[23] W.-H. Liao and P.-M. Chen, "Analysis of visual elements in logo design," in Proceedings of the 12th International Symposium on Smart Graphics, pp. 73-85, Springer, Switzerland, August 2014.

[24] Q. Wang, "Statistical analysis on the enterprise logo color designs of global 500," in Proceedings of the EEE 10th International Conference on Computer-Aided Industrial Design and Conceptual Design, pp. 277-280, Wenzhou, China, November 2009.

[25] B. Saleh, M. Dontcheva, A. Hertzmann, and Z. Liu, "Learning style similarity for searching infographics," in Proceedings of the Graphics Interface Conference, pp. 59-64, Canadian Information Processing Society, Toronto, Canada, May 2015.

[26] F. Atneave, "Some informational aspects of visual perception," Psychological Review, vol. 61, no. 3, pp. 183-193, 1954.

[27] I. Lazakis, C. Gkerekos, and G. Theotokatos, "Investigating an SVM-driven, one-class approach to estimating ship systems condition," Ships and Offshore Structures, vol. 14, no. 5, pp. 432-441, 2019.

[28] Y. Peng, A. Rios, R. Kavuluru, and Z. Lu, "Extracting chemical-protein relations with ensembles of SVM and deep learning models," Database, vol. 2018, 2018. 